\title{
La flore lactique du fromage bleu de Cabrales
}

\author{
par \\ M. NUÑEZ et Margarita MEDINA \\ Departamento de Bioquímica y Microbiología. CRIDA 06 \\ Instituto Nacional de Investigaciones Agrarias \\ Apdo, 8111 Madrid
}

\section{INTRODUCTION}

Le fromage de Cabrales est le plus réputé des fromages persillés produits en Espagne. Il est fabriqué à partir de lait cru de vache, mélangé avec des petites quantités de lait de brebis et de chèvre, sans utiliser de levains lactiques ou fongiques. On a suivi l'évolution de sa flore microbienne (Nunez, 1978) et on a constaté que les streptocoques lactiques sont les micro-organismes dominants en surface et à l'intérieur du fromage pendant la fabrication, le salage ( $3^{\mathrm{e}}$ et $4^{\mathrm{e}} \mathrm{j}$ ) et le premier affinage ( $\mathrm{du} 5^{\mathrm{e}}$ au $\left.15^{\mathrm{e}} \mathrm{j}\right)$. Le deuxième affinage se poursuit pendant 3 à 4 mois en grottes naturelles dont la température est de 9 à $10^{\circ} \mathrm{C}$ et l'humidité de 90 à $95 \mathrm{p} .100$ : après le $30^{\mathrm{e}} \mathrm{j}$ de maturation les lactobacilles, les levures et les moisissures dominent à l'intérieur du fromage et les microcoques en surface.

La flore lactique du fromage de Roquefort est bien connue : Devoyod et Muller (1969) ont étudié les streptocoques lactiques et les leuconostocs et il y a aussi des travaux publiés sur les entérocoques (Devoyod, 1969) et les lactobacilles (Devoyod, 1970). En ce qui concerne le fromage de Cabrales, les lactobacilles sont le seul genre des bactéries lactiques qui a fait l'objet d'une étude (Burgos et al., 1971). Ces auteurs ont mentionné Lactobacillus casei comme l'espèce dominante sur 6 souches isolées de lactobacilles.

$\mathrm{Au}$ cours du présent travail nous avons recherché à identifier les principales espèces des bactéries lactiques trouvées dans le fromage de Cabrales et étudié quelques caractères physiologiques qui peuvent présenter un intérêt en technologie fromagère. 


\section{MATERIEL ET METHODES}

\section{Dénombrements et isolements des souches}

Nous avons suivi l'évolution de la flore lactique au cours de deux fabrications traditionnelles (A et B) de fromage de Cabrales. La méthode de fabrication du fromage et les techniques et milieux utilisés pour les dénombrements des streptocoques, leuconostocs et lactobacilles ont été décrits précédemment (Nunez, 1978.)

Les échantillons de lait ont été prélevés avant l'emprésurage, les échantillons de caillé avant la mise en moules et les échantillons de la surface et de l'intérieur du fromage les $4^{\mathrm{e}}, 15^{\mathrm{e}}, 30^{\mathrm{e}}, 60^{\mathrm{e}}, 90^{\mathrm{e}}$ et $120^{\mathrm{e}} \mathrm{j}$ après l'emprésurage. Pour chaque échantillon, 4 à 10 colonies à partir de chacun des milieux ont été repiquées sur gélose M.R.S. (De Man et al., 1960). Après purification, les souches étaient conservées à $-30^{\circ} \mathrm{C}$ sur bouillon M.R.S. $(0,5 \mathrm{ml}$ de culture de $16 \mathrm{~h}$ sur $4,5 \mathrm{ml}$ de bouillon).

\section{Identification des souches}

Pour séparer les différents genres des bactéries lactiques on a d'abord étudié les caractères suivants :

\section{Morphologie}

L'examen microscopique des souches isolées et leur coloration de Gram ont été effectués sur une culture de $16 \mathrm{~h}$ à $30^{\circ} \mathrm{C}$ sur bouillon M.R.S.

\section{Production de catalase}

Une goutte de $\mathrm{H}_{2} \mathrm{O}_{2}$ à 10 volumes était déposée sur une colonie de $48 \mathrm{~h}$ sur gélose M.R.S.

\section{Production de $\mathrm{CO}_{2}$ à partir du glucose}

On l'a observée sur bouillon M.R.S. avec bouchon de gélose double incubé $7 \mathrm{j}$ à $30^{\circ} \mathrm{C}$.

\section{Désamination de l'arginine}

Le bouillon d'Elliker et al. (1956) additionné de L-monochlorhydrate d'arginine à 0,3 p. 100 de concentration finale a été utilisé. La lecture du résultat se faisait en ajoutant une goutte de réactif de Nessler à une goutte de culture de $72 \mathrm{~h}$ à $30^{\circ} \mathrm{C}$.

On a de suite identifié les souches appartenant aux trois genres trouvés, d'après le Bergey's Manual of Determinative Bacteriology (Buchanan et Gibbons, 1974), par l'examen des caractères suivants : 


\section{a) StREPTOCOQUeS}

\section{Croissance à différentes températures}

Le développement bactérien sur bouillon d'Elliker était apprécié après $48 \mathrm{~h}$ pour les cultures incubées à $40^{\circ} \mathrm{C}, 45^{\circ} \mathrm{C}$ et $50^{\circ} \mathrm{C}$ et après $7 \mathrm{j}$ pour les cultures incubées à $10^{\circ} \mathrm{C}$.

\section{Croissance en présence de 0,1 p.100 de bleu de méthylène}

On a utilisé de la poudre de lait écrémé reconstitué à 10 p. 100 à l'aide de l'eau distillée et additionnée de bleu de méthylène à une concentration finale de 0,1 p. 100.

3. Croissance en présence de 4 p. 100 et 6,5 p. 100 de $\mathrm{NaCl}$

Les souches étaient ensemencées dans le bouillon d'Elliker additionné de $\mathrm{NaCl}$ (concentrations finales : 4 p. 100 et 6,5 p. 100) et examinées après $48 \mathrm{~h}$ à $30^{\circ} \mathrm{C}$.

4. Croissance en présence de 0,04 p.100 de tellurite de potassium

Le milieu Tryptose Blood Agar Base (Difco) contenant 0,04 p. 100 de tellurite de potassium (concentration finale) coulé en boîtes de Pétri et ensemencé en surface a été utilisé.

\section{Croissance à $p H \quad 9,6$}

Les souches étaient cultivées à $30^{\circ} \mathrm{C}$ sur le milieu de Shattock et Hirsch (1947) et examinées après $24 \mathrm{~h}$.

6. Résistance à un chauffage de $60^{\circ} \mathrm{C}$ pendant $30 \mathrm{mn}$

La méthode décrite par Sandine et al. (1962) a été utilisée.

\section{Action sur le lait tournesolé}

La réduction, acidification et coagulation étaient notées après $7 \mathrm{j}$ d'incubation à $30^{\circ} \mathrm{C}$.

\section{Hydrolyse de la gélatine}

La recherche de l'hydrolyse de la gélatine a été faite selon la méthode de Frazier (1926).

\section{Production de $\mathrm{CO}_{2}$ à partir du citrate}

Le milieu utilisé était du bouillon de Reddy et al. (1971). Les cultures ont été incubées $72 \mathrm{~h}$ à $30^{\circ} \mathrm{C}$.

\section{Production de diacétyle à partir du glucose}

La production de diacétyle à partir du glucose était décelée selon la méthode de Barritt (1936), après $48 \mathrm{~h}$ et $7 \mathrm{j}$ d'incubation à $30^{\circ} \mathrm{C}$ sur un bouillon de composition : peptone $1 \mathrm{p} .100$; glucose 0,5 p. 100 ; pH 7,0. 


\section{Fermentation des sucres}

Les souches étaient cultivées pendant $14 \mathrm{j}$ à $30^{\circ} \mathrm{C}$ sur un bouillon de composition : peptone 1 p. 100 ; $\mathrm{NaCl} 0,5$ p. 100 ; pourpre de bromocrésol 0,004 p. 100 ; pH 7,0. La concentration finale en hydrates de carbone était de 0,5 p. 100.

\section{Activité acidifiante}

La mesure de l'activité acidifiante a été faite selon la méthode décrite par Devoyod et Muller (1969).

\section{b) LeUconostocs}

\section{Production de dextrane}

Le milieu de Garvie (1960) a été utilisé. La lecture du résultat se faisait après $5 \mathrm{j}$ d'incubation à $22^{\circ} \mathrm{C}$.

\section{Hydrolyse de l'esculine}

Le bouillon de Naylor et Sharpe (1958) était incubé 7 j à 22。 C. On décelait l'hydrolyse de l'esculine par le noircissement du milieu et par la perte de fluorescence en lumière ultra-violette.

\section{Production de diacétyle à partir du citrate}

Après $48 \mathrm{~h}$ et $7 \mathrm{j}$ d'incubation à $22^{\circ} \mathrm{C}$ sur bouillon d'Elliker additionné de citrate trisodique (concentration finale : 0,5 p. 100) la présence du diacétyle était décelée selon Barritt (1936).

4. Croissance à $p H$ initial 4,8 et 6,5

On a suivi la méthode décrite par Garvie (1967).

5. Croissance en présence de 3 p. 100 et 6,5 p. 100 de $\mathrm{NaCl}$

Le bouillon d'Elliker additionné de $\mathrm{NaCl}$ (concentrations finales 3 p. 100 et 6,5 p. 100) était incubé $3 \mathrm{j}$ à $22^{\circ} \mathrm{C}$.

\section{Croissance à $37^{\circ} \mathrm{C}$}

Le développement bactérien sur bouillon d'Elliker était observé après $3 \mathrm{j}$ à $37^{\circ} \mathrm{C}$.

\section{Fermentation des sucres}

La fermentation des sucres a été faite selon la méthode décrite par Garvie (1967).

\section{c) LACTOBACILLES}

\section{Croissance à différentes températures}

Le développement des souches sur bouillon M.R.S. était noté après $7 \mathrm{j}$ d'incubation pour les cultures à $45^{\circ} \mathrm{C}$ et après $14 \mathrm{j}$ pour les cultures à $15^{\circ} \mathrm{C}$. 


\section{Production de diacétyle à partir du citrate}

On a suivi la méthode décrite ci-dessus pour les leuconostocs.

3. Hydrolyse de l'esculine nostocs.

On a suivi la méthode décrite préalablement pour les leuco-

\section{Fermentation des sucres}

Le milieu utilisé était le bouillon M.R.S. exempt de glucose et d'extrait de viande avec 0,004 p. 100 de rouge de chlorophénol et une concentration finale en hydrates de carbone de 2 p. 100. Les cultures ont été incubées $14 \mathrm{j}$ à $30^{\circ} \mathrm{C}$.

\section{Acidité titrable}

L'acidité produite pendant $7 \mathrm{j}$ d'incubation à $30^{\circ} \mathrm{C}$ sur lait écrémé reconstitué à 10 p. 100 était titrée sur $9 \mathrm{~g}$ de culture à l'aide de $\mathrm{NaOH}$ $0,1 \mathrm{~N}$ en présence de phénolphtaléine.

Croissance des bactéries lactiques à différentes concentrations de chlorure de sodium

Les temps de génération de 12 streptocoques, 12 leuconostocs et 12 lactobacilles sur bouillon M.R.S. additionné de $\mathrm{NaCl}$ à différentes concentrations ont été déterminés à l'aide d'un biophotomètre enregistreur Jobin-Yvon.

\section{RESULTATS}

\section{Identification de la flore lactique}

Lors de notre étude, 261 souches de bactéries lactiques ont été isolées du lait, du caillé et du fromage de Cabrales au cours de l'affinage. Parmi ces souches, 85 ont été identifiées comme étant des streptocoques, 59 comme des leuconostocs et 117 comme des lactobacilles. Nous n'avons pas mis en évidence la présence de pédiocoques. La répartition des différentes espèces de bactéries lactiques isolées des deux fabrications est indiquée dans le tableau 1.

\section{a) CARACtères biochimiques des Streptocoques}

Les caractères biochimiques des streptocoques sont donnés dans le tableau 2. Cinquante-quatre souches de streptocoques correspondaient à la description de l'espèce Streptococcus lactis : ces bactéries se développaient à $10^{\circ} \mathrm{C}$ et à $40^{\circ} \mathrm{C}$ et en présence de $0,1 \mathrm{p} .100 \mathrm{de}$ bleu de méthylène et de 4 p. 100 de $\mathrm{NaCl}$, désaminaient l'arginine et fermentaient le maltose et le lactose; elles ne poussaient pas à $45^{\circ} \mathrm{C}$, à $\mathrm{pH} 9,6$ ni en présence de $6,5 \mathrm{p} .100$ de $\mathrm{NaCl}$ et ne produisaient pas du $\mathrm{CO}_{2}$ à partir du citrate ni du diacétyle à partir du glucose. 


\section{TABLEAU 1}

Répartition des bactéries lactiques isolées du fromage de Cabrales

\begin{tabular}{|c|c|c|c|c|c|c|}
\hline \multirow{2}{*}{ Espèce } & \multicolumn{3}{|c|}{ Fabrication A } & \multicolumn{3}{|c|}{ Fabrication B } \\
\hline & $\mathrm{L}+\mathrm{C}$ & I & $\mathrm{E}$ & $L+C$ & $\mathrm{I}$ & $\mathrm{E}$ \\
\hline Streptococcus lactis & 12 & 12 & 5 & 11 & 4 & 10 \\
\hline Str. lactis atypiques & 3 & 5 & - & 3 & 7 & 1 \\
\hline Str. faecium & - & - & 1 & - & 3 & - \\
\hline Str. faecalis & - & - & - & - & - & 1 \\
\hline Str. acidominimus & - & - & 2 & - & - & - \\
\hline Str. acidominimus atypiques & - & - & 5 & - & - & - \\
\hline Leuconostoc dextranicum & - & 3 & 1 & 6 & 7 & 10 \\
\hline L. lactis & - & - & 1 & 3 & 1 & 1 \\
\hline L. mesenteroides & 2 & 2 & 1 & 1 & 1 & 1 \\
\hline L. paramesenteroides & 2 & 4 & 7 & 4 & 1 & - \\
\hline Lactobacillus plantarum & 13 & 27 & 17 & 6 & 28 & 15 \\
\hline L. plantarum atypiques & - & 2 & - & - & - & - \\
\hline L. casei subsp. casei & - & - & 2 & - & - & 4 \\
\hline L. casei subsp. rhamnosus & - & - & - & - & 1 & 1 \\
\hline L. brevis & - & 1 & - & - & - & - \\
\hline
\end{tabular}

L + C : lait + caillé ; I : intérieur ; E : extérieur.

Dix-neuf souches de streptocoques lactiques ont été considérées comme des souches atypiques de l'espèce Str. lactis : deux souches ne produisaient pas d'acide à partir du maltose; une souche ne se développait pas à $40^{\circ} \mathrm{C}$; une autre souche ne poussait pas en présence de 0,1 p. 100 de bleu de méthylène; trois souches ne produisaient pas de l'ammoniaque à partir de l'arginine; 6 souches ne désaminaient pas l'arginine et ne se développaient pas en présence de 0,1 p. 100 de bleu de méthylène; deux souches se développaient en présence de 6,5 p. 100 de $\mathrm{NaCl}$, ne désaminaient pas l'arginine et ne se développaient pas en présence de 0,1 p. 100 de bleu de méthylène; 4 souches se développaient à $\mathrm{pH} 9,6$. Les autres caractères biochimiques correspondaient à ceux de Str. lactis.

Cinq souches d'entérocoques ont été isolées sur le milieu de Kenner et al. (1961), où ils sont trouvés occasionnellement en faible nombre $\left(10^{2}-10^{3} / \mathrm{g}\right.$ de fromage) au cours de la maturation. Les 5 souches se développaient à $10^{\circ} \mathrm{C}$, à $45^{\circ} \mathrm{C}$ et à $\mathrm{pH} 9,6$, poussaient en présence de 0,1 p. 100 de bleu de méthylène et de 6,5 p. 100 de $\mathrm{NaCl}$, 


\section{TABLEAU 2}

Caractères biochimiques des streptocoques isolés du fromage de Cabrales

\begin{tabular}{|c|c|c|c|c|c|c|}
\hline Espèce & $\begin{array}{c}\text { Str. } \\
\text { lactis }\end{array}$ & $\begin{array}{l}\text { Str. } \\
\text { lactis } \\
\text { atypi- } \\
\text { ques }\end{array}$ & $\begin{array}{c}\text { Str. } \\
\text { fae- } \\
\text { cium }\end{array}$ & $\begin{array}{l}\text { Str. } \\
\text { fae- } \\
\text { calis }\end{array}$ & $\begin{array}{l}\text { Str. } \\
\text { acido- } \\
\text { mini- } \\
\text { mus }\end{array}$ & $\begin{array}{c}\text { Str. } \\
\text { acido- } \\
\text { mini- } \\
\text { mus } \\
\text { atypi- } \\
\text { ques }\end{array}$ \\
\hline Nombre de souches & 54 & 19 & 4 & 1 & 2 & 5 \\
\hline Croissance à $10^{\circ} \mathrm{C}$ & + & + & + & + & - & - \\
\hline Croissance à $40^{\circ} \mathrm{C}$ & + & $18 / 19$ & + & + & - & - \\
\hline Croissance à $45^{\circ} \mathrm{C}$ & - & - & + & + & - & - \\
\hline Croissance à pH 9,6 & - & $4 / 19$ & + & + & - & - \\
\hline Croissance 4 p. $100 \mathrm{NaCl}$ & + & + & + & + & + & + \\
\hline Croissance 6,5 p. $100 \mathrm{NaCl}$ & - & $2 / 19$ & + & + & - & - \\
\hline Croissance 0,1 p. 100 B.M. & + & $6 / 19$ & + & + & - & - \\
\hline Croissance 0,04 p. 100 tellurite & N.E. & N.E. & - & + & N.E. & N.E. \\
\hline Résistance $60^{\circ} \mathrm{C} / 30 \mathrm{mn}$ & $50 / 54$ & $17 / 19$ & + & + & + & $3 / 5$ \\
\hline A.R.C. lait tournesolé & $48 / 54$ & $17 / 19$ & + & + & - & - \\
\hline Hydrolyse de la gélatine & N.E. & N.E. & - & - & N.E. & N.E. \\
\hline Désamination de l'arginine & + & $4 / 19$ & + & + & - & - \\
\hline Gaz à partir du citrate & - & - & - & - & - & $1 / 5$ \\
\hline Diacétyle à partir du glucose & - & - & - & - & - & - \\
\hline Fermentation de l'arabinose & $12 / 54$ & $11 / 19$ & $2 / 4$ & + & - & $3 / 5$ \\
\hline Fermentation du lactose & + & + & + & + & - & - \\
\hline Fermentation du maltose & + & $17 / 19$ & + & + & + & $4 / 5$ \\
\hline Fermentation du mannitol & $23 / 54$ & $15 / 19$ & $2 / 4$ & + & + & $2 / 5$ \\
\hline Fermentation du mélézitose & $17 / 54$ & $12 / 19$ & - & + & + & $2 / 5$ \\
\hline Fermentation du mélibiose & $3 / 54$ & $13 / 19$ & $2 / 4$ & - & + & $1 / 5$ \\
\hline Fermentation du sorbitol & $2 / 54$ & $10 / 19$ & - & + & - & - \\
\hline
\end{tabular}

N.E. : non essayée.

B.M. : bleu de méthylène.

A.R.C. : acidification, réduction et coagulation.

Fraction: nombre de souches positives / nombre de souches essayées.

produisaient de l'ammoniaque à partir de l'arginine et résistaient à un chauffage de $60^{\circ} \mathrm{C}$ pendant $30 \mathrm{mn}$. Elles ne produisaient pas du $\mathrm{CO}_{2}$ à partir du citrate ni du diacétyle à partir du glucose. Quatre souches ont été identifiées comme appartenant à l'espèce Str. fae cium : elles ne se développaient pas à $50^{\circ} \mathrm{C}$ ni en présence de 0,04 p. 100 de tellurite de potassium et ne produisaient pas d'acide à partir du mélézitose ni du sorbitol. La souche restante correspondait à la description de Str. faecalis. Elle n'hydrolysait pas la gélatine. 
Deux souches correspondaient à la description de Str. acidominimus : elles ne poussaient pas à $10^{\circ} \mathrm{C}$ ni à $45^{\circ} \mathrm{C}$, ne désaminaient pas l'arginine, ne se développaient pas en présence de 0,1 p. 100 de bleu de méthylène, de 6,5 p. 100 de $\mathrm{NaCl}$ ni à $\mathrm{pH} 9,6$, n'étaient pas hémolytiques et ne fermentaient pas le glycérol. Cinq souches ont été considérées comme des souches atypiques de l'espèce Str. acidominimus : elles n'hydrolysaient pas l'hippurate.

Les 7 souches de Str. acidominimus ont été isolées à la surface du fromage A le $90^{\mathrm{e}}$ et le $120^{\mathrm{e}} \mathrm{j}$ après l'emprésurage. Leur $\mathrm{pH}$ final dans bouillon glucosé était 5,50-6,15.

Les activités acidifiantes des streptocoques sont indiquées dans le tableau 3. Les 8 souches les plus acidifiantes ont été isolées de la fabrication $\mathrm{B}$, à partir du lait, du caillé et du fromage pendant le premier mois d'affinage.

TABLEAU 3

Activité acidifiante des streptocoques isolés du fromage de Cabrales

\begin{tabular}{|c|c|c|c|c|c|}
\hline \multirow{2}{*}{ Espèce } & \multirow{2}{*}{$\begin{array}{l}\text { Nombre } \\
\text { de } \\
\text { souches }\end{array}$} & \multicolumn{4}{|c|}{ Acidité après $6 \mathrm{~h}$ à $30^{\circ} \mathrm{C}$} \\
\hline & & $\leqslant 20^{\circ} \mathrm{D}$ & $21-25^{\circ} \mathrm{D}$ & $26-30^{\circ} \mathrm{D}$ & $31-35^{\circ} \mathrm{D}$ \\
\hline Streptococcus lactis & 54 & - & 28 & 19 & 7 \\
\hline Str. lactis atypiques & 19 & - & 15 & 3 & 1 \\
\hline Str. faecium & 4 & - & 4 & - & - \\
\hline Str. faecalis & 1 & - & 1 & - & - \\
\hline Str. acidominimus & 2 & 2 & - & - & - \\
\hline Str. acidominimus atypiques & 5 & 5 & - & - & - \\
\hline
\end{tabular}

\section{b) Caractères BIOchimiques DES LeUconostocs}

Les caractères biochimiques des leuconostocs sont donnés dans le tableau 4. Vingt-sept souches sur les 59 isolées peuvent être considérées comme appartenant à l'espèce Leuconostoc dextranicum : elles produisaient des dextranes à partir du saccharose, fermentaient le lactose et ne fermentaient pas l'arabinose. La majeure partie de ces 
TABLEAU 4

Caractères biochimiques des leuconostocs isolés du fromage de Cabrales

\begin{tabular}{|c|c|c|c|c|}
\hline Espèce & $\begin{array}{l}\text { L. dextra- } \\
\text { nicum }\end{array}$ & $\begin{array}{l}\text { L. mesen- } \\
\text { teroides }\end{array}$ & $\begin{array}{l}\text { L. parame } \\
\text { senteroi- } \\
\text { des }\end{array}$ & L. lactis \\
\hline $\begin{array}{l}\text { Nombre de souches } \\
\text { Gaz à partir du glucose } \\
\text { Désamination de l'arginine } \\
\text { Production de dextrane } \\
\text { Croissance à } 37^{\circ} \mathrm{C} \\
\text { Croissance } 3 \mathrm{p} .100 \mathrm{NaCl} \\
\text { Croissance } 6,5 \mathrm{p} .100 \mathrm{NaCl} \\
\text { Croissance pH initial } 4,8 \\
\text { Croissance pH initial } 6,5 \\
\text { Diacétyle à partir du citrate } \\
\text { Hydrolyse de l'esculine } \\
\text { Fermentation de l'arabinose } \\
\text { Fermentation du glucose } \\
\text { Fermentation du lactose } \\
\text { Fermentation du maltose } \\
\text { Fermentation du saccharose } \\
\text { Fermentation de la salicine } \\
\text { Fermentation du tréhalose }\end{array}$ & $\begin{array}{c}27 \\
+ \\
- \\
+ \\
+ \\
+ \\
- \\
9 / 27 \\
+ \\
- \\
2 / 27 \\
- \\
+ \\
+ \\
22 / 27 \\
26 / 27 \\
5 / 27 \\
26 / 27\end{array}$ & $\begin{array}{l}8 \\
+ \\
- \\
+ \\
+ \\
+ \\
+1 / 8 \\
+ \\
+ \\
+ \\
+ \\
+ \\
+ \\
+ \\
+ \\
+ \\
+7 / 8\end{array}$ & $\begin{array}{c}18 \\
+ \\
- \\
- \\
+ \\
+ \\
4 / 18 \\
4 / 18 \\
+ \\
- \\
2 / 18 \\
1 / 18 \\
+ \\
+ \\
+ \\
16 / 18 \\
2 / 18 \\
+\end{array}$ & $\begin{array}{l}6 \\
+ \\
- \\
\overline{-} \\
+ \\
= \\
+ \\
+ \\
- \\
+ \\
+ \\
+ \\
+ \\
+\end{array}$ \\
\hline
\end{tabular}

Fraction : nombre de souches positives / nombre de souches essayées.

souches produisaient de l'acide à partir du maltose, du saccharose et du tréhalose, ne fermentaient pas la salicine et n'hydrolysaient pas l'esculine.

Huit souches correspondaient à la description de l'espèce $L$. mesenteroides : elles produisaient des dextranes, hydrolysaient l'esculine et fermentaient l'arabinose, le lactose, le maltose, le saccharose, la salicine et le tréhalose.

Dix-huit souches ont été identifiées comme $L$. paramesenteroides : elles ne formaient pas des dextranes, ne produisaient pas du diacétyle et fermentaient le lactose, le maltose, le saccharose et le tréhalose.

Les 6 souches restantes appartenaient à l'espèce L. lactis : elles ne formaient pas des dextranes, ne produisaient pas du diacétyle et fermentaient le lactose, le maltose et le saccharose. 


\section{c) CARACTÈRES BIOchimiques des LACTOBACILLES}

Les caractères biochimiques des lactobacilles sont indiqués dans le tableau 5. Cent-six souches correspondaient à la description de Lactobacillus plantarum : elles hydrolysaient l'esculine et produisaient de l'acide à partir du cellobiose, du lactose, du maltose, du mélézitose, du mélibiose, du raffinose, du ribose, du saccharose et de la salicine. Deux souches ont été considérées comme L. plantarum atypiques : elles fermentaient le mélibiose et le raffinose mais ne fermentaient pas le cellobiose, le mélézitose, le saccharose et la salicine.

Six souches ont les caractères de L. casei subsp. casei : elles produisaient de l'acide à partir du cellobiose, du lactose, du maltose,

\section{TABLEAU 5}

Caractères biochimiques des lactobacilles isolés du fromage de Cabrales

Nombre de souches

Croissance à $15^{\circ} \mathrm{C}$

Croissance à $45^{\circ} \mathrm{C}$

Gaz à partir du glucose

Désamination de l'arginine

Diacétyle à partir du citrate

Hydrolyse de l'esculine

Fermentation du cellobiose

Fermentation du glucose

Fermentation du lactose

Fermentation du maltose

Fermentation du mélézitose

Fermentation du mélibiose

Fermentation du raffinose

Fermentation du rhamnose

Fermentation du ribose

Fermentation du saccharose

Fermentation de la salicine

Fermentation du xylose

\begin{tabular}{|c|c|c|c|c|}
\hline $\begin{array}{l}\text { L. } \\
\text { planta- } \\
\text { rum }\end{array}$ & $\begin{array}{l}\text { L. } \\
\text { planta- } \\
\text { rum } \\
\text { atypi- } \\
\text { ques }\end{array}$ & $\begin{array}{l}\text { L. casei } \\
\text { subsp. } \\
\text { casei }\end{array}$ & $\begin{array}{l}\text { L. casei } \\
\text { subsp. } \\
\text { rhamno- } \\
\text { sus }\end{array}$ & L. brevis \\
\hline 106 & 2 & 6 & 2 & 1 \\
\hline+ & + & + & + & + \\
\hline $85 / 106$ & - & - & - & - \\
\hline - & - & - & - & + \\
\hline$\overline{60}$ & - & $\overline{1 / 6}$ & - & + \\
\hline $\begin{array}{c}69 / 106 \\
+\end{array}$ & $\overline{+}$ & $\begin{array}{c}1 / 6 \\
+\end{array}$ & $\bar{t}$ & - \\
\hline+ & - & + & $1 / 2$ & - \\
\hline+ & + & + & + & + \\
\hline+ & + & + & + & + \\
\hline+ & + & + & + & + \\
\hline+ & - & + & $1 / 2$ & - \\
\hline+ & + & - & - & + \\
\hline+ & + & - & - & - \\
\hline $35 / 106$ & - & - & + & - \\
\hline+ & + & + & + & + \\
\hline+ & - & $4 / 6$ & - & - \\
\hline+ & - & $5 / 6$ & $1 / 2$ & - \\
\hline - & + & - & $1 / 2$ & + \\
\hline
\end{tabular}

Fraction : nombre de souches positives / nombre de souches essayées. 
du mélézitose, du ribose et la majeure partie du saccharose et de la salicine. Deux souches peuvent être considérées comme appartenant à $L$. casei subsp. rhamnosus : elles fermentaient le rhamnose. Une souche a été classée comme L. brevis : elle produisait du $\mathrm{CO}_{2}$ à partir du glucose, désaminait l'arginine et fermentait le lactose, le maltose, le mélibiose, le ribose et le xylose.

L'acidité titrable des lactobacilles est donnée dans le tableau 6. La majeure partie des souches de L. plantarum peu acidifiantes $(32 / 38$ soit 84 p. 100$)$ ont été isolées du fromage B.

\section{TABLEAU 6}

Acidité titrable des lactobacilles isolés du fromage de Cabrales

\begin{tabular}{|c|c|c|c|c|c|c|}
\hline \multirow{2}{*}{ Espèce } & \multirow{2}{*}{$\begin{array}{l}\text { Nombre } \\
\text { de } \\
\text { souches }\end{array}$} & \multicolumn{5}{|c|}{ Acidité $\left({ }^{\circ} \mathrm{D}\right)$ après $7 \mathrm{j}$ à $30^{\circ} \mathrm{C}$} \\
\hline & & $20-50$ & $51-80$ & $81-110$ & $111-140$ & $141-170$ \\
\hline L. plantarum & 106 & 38 & 5 & 41 & 17 & 5 \\
\hline L. plantarum atypiques & 2 & 2 & - & - & - & - \\
\hline L. casei subsp. casei & 6 & 2 & 2 & - & 1 & 1 \\
\hline L. casei subsp. rhamnosus & 2 & 1 & 1 & - & - & - \\
\hline L. brevis & 1 & 1 & - & - & - & - \\
\hline
\end{tabular}

d) CROISSANCE DES BACTÉRIES LACTIQUES A DIFFÉRENTES CONCENTRATIONS DE CHLORURE DE SODIUM

Nous avons déterminé les temps de génération de 36 souches de bactéries lactiques, 18 isolées au centre du fromage et 18 isolées à la surface, en bouillon M.R.S. additionné de $\mathrm{NaCl}$ à différentes concentrations. Sur le tableau 7 , on a indiqué la moyenne des temps de génération pour les souches appartenant à chaque espèce en présence des différentes concentrations de $\mathrm{NaCl}$. 
TABLEAU 7

Temps de génération des principales espèces de bactéries lactiques isolées du fromage de Cabrales en présence de différentes concentrations de $\mathrm{NaCl}$

\begin{tabular}{|c|c|c|c|c|c|c|c|c|}
\hline \multirow{2}{*}{ Espèce } & \multirow{2}{*}{$\begin{array}{c}\text { Nombre } \\
\text { de } \\
\text { souches }\end{array}$} & \multicolumn{6}{|c|}{$\begin{array}{c}\text { Moyenne des temps de génération }(\mathrm{mn}) \text { à différentes } \\
\text { concentrations de } \mathrm{NaCl}\end{array}$} & \multirow{2}{*}{$\begin{array}{l}\text { Rapport } \\
5 \text { p. } 100 / \\
0 \text { p. } 100\end{array}$} \\
\hline & & 0 p. 100 & 1 p. 100 & 2 p. 100 & 3 p. 100 & 4 p. 100 & 5 p. 100 & \\
\hline \multicolumn{9}{|l|}{ Intérieur : } \\
\hline $\begin{array}{l}\text { Str. lactis } \\
\text { Str. faecium } \\
\text { Leuc. dextranicum } \\
\text { Leuc. paramesenteroides } \\
\text { Leuc. lactis } \\
\text { Lb. plantarum }\end{array}$ & $\begin{array}{l}5 \\
1 \\
4 \\
1 \\
1 \\
6\end{array}$ & $\begin{array}{r}76,2 \\
76,5 \\
129,5 \\
105,0 \\
141,0 \\
111,5\end{array}$ & $\begin{array}{r}106,6 \\
96,0 \\
118,7 \\
87,0 \\
111,0 \\
111,0\end{array}$ & $\begin{array}{r}108,0 \\
103,5 \\
138,5 \\
78,0 \\
210,0 \\
95,8\end{array}$ & $\begin{array}{l}109,6 \\
101,0 \\
174,5 \\
102,0 \\
300,0 \\
109,0\end{array}$ & $\begin{array}{l}152,1 \\
120,0 \\
204,5 \\
133,0 \\
387,0 \\
126,1\end{array}$ & $\begin{array}{l}255,4 \\
180,0 \\
289,7 \\
163,0 \\
591,0 \\
223,5\end{array}$ & $\begin{array}{l}3,35 \\
2,35 \\
2,24 \\
1,55 \\
4,19 \\
2,00\end{array}$ \\
\hline \multicolumn{9}{|l|}{ Extérieur : } \\
\hline $\begin{array}{l}\text { Str. lactis } \\
\text { Str. faecalis } \\
\text { Leuc. dextranicum } \\
\text { Leuc. lactis } \\
\text { Lb. plantarum } \\
\text { Lb. casei }\end{array}$ & $\begin{array}{l}5 \\
1 \\
4 \\
2 \\
3 \\
3\end{array}$ & $\begin{array}{r}72,9 \\
76,5 \\
101,2 \\
91,5 \\
97,0 \\
163,0\end{array}$ & $\begin{array}{r}96,6 \\
81,0 \\
79,7 \\
75,0 \\
90,3 \\
147,0\end{array}$ & $\begin{array}{r}106,8 \\
91,5 \\
118,0 \\
108,0 \\
79,0 \\
172,0\end{array}$ & $\begin{array}{r}135,3 \\
84,0 \\
149,5 \\
132,0 \\
99,0 \\
209,0\end{array}$ & $\begin{array}{r}190,4 \\
117,0 \\
226,7 \\
340,5 \\
99,0 \\
231,3\end{array}$ & $\begin{array}{l}409,0 \\
174,0 \\
271,5 \\
531,0 \\
187,0 \\
443,3\end{array}$ & $\begin{array}{l}5,61 \\
2,27 \\
2,68 \\
5,80 \\
1,93 \\
2,72\end{array}$ \\
\hline
\end{tabular}




\section{DISCUSSION}

\section{a) LES STREPTOCOQUES}

Nos résultats ont montré que Streptococcus lactis est l'espèce dominante des streptocoques lactiques et du genre Streptococcus dans le fromage de Cabrales. L'espèce Str. cremoris n'a pas été isolée, contrairement à ce que Devoyod et Muller (1969) ont trouvé dans le fromage de Roquefort où elle représente 24 p. 100 des streptocoques lactiques. Pourtant, nous avons identifié 19 souches de streptocoques lactiques avec quelques caractères de Str. cremoris mais qui ont été considérées comme des souches atypiques de Str. Lactis. Cavett et Garvie (1967) et Orvin Mundt (1976) ont étudié aussi des souches atypiques de Str. lactis.

Les souches de streptocoques lactiques isolées du fromage de Cabrales sont en général des germes d'acidification lente : seulement $8 / 73$ soit 11 p. 100 , produisaient plus de $30^{\circ} \mathrm{D}$ en $6 \mathrm{~h}$.

En ce qui concerne les entérocoques, nous avons constaté qu'ils sont rencontrés occasionnellement à la surface et au centre du fromage, Str. faecium étant l'espèce dominante. Leur faible nombre restreint leur action stimulante vis-à-vis de la croissance des streptocoques lactiques et des leuconostocs montrée par Devoyod et Muller (1969) et Devoyod et Desmazeaud (1970).

Nous croyons que c'est la première fois qu'on isole Str. acidominimus à partir d'un fromage. Cependant il avait déjà été isolé à partir du lait cru.

D'après nos résultats (tab. 7), les streptocoques étaient, parmi les bactéries lactiques, le genre le plus sensible au chlorure de sodium si on considère le rapport « temps de génération avec $5 \mathrm{p} .100$ $\mathrm{NaCl} /$ temps de génération avec 0 p. $100 \mathrm{NaCl} »$ : il était en moyenne de 3,18 pour 6 souches de l'intérieur du fromage et de 5,03 pour 6 souches de l'extérieur.

Str. lactis était l'espèce la moins résistante : le rapport est en moyenne de 3,35 pour 5 souches de l'intérieur et de 5,61 pour 5 souches de l'extérieur, contrairement à ce qu'on pouvait attendre compte tenu de la forte teneur en chlorure de sodium à la surface du fromage. Les entérocoques ont les rapports les plus bas : 2,35 pour Str. faecium et 2,27 pour Str. faecalis, conformément à leur halotolérance.

\section{b) LES LEUCONOSTOCS}

Les espèces les plus fréquemment retrouvées étaient Leuconostoc dextranicum et $L$. paramesenteroides, ce qui coïncide avec les résultats signalés pour un autre fromage espagnol, le Manchego, par Nunez (1976). Seulement 10 p. 100 des souches ont été identifiées comme L. lactis et l'espèce $L$. cremoris n'a pas été isolée. Ces données 
sont analogues à celles obtenues par Devoyod et Muller (1969) pour le fromage de Roquefort.

Aucune des 59 souches de leuconostocs étudiées n'était capable de produire du diacétyle à partir du citrate. En outre, la population de leuconostocs à l'intérieur du fromage reste toujours inférieure à $10^{7} / \mathrm{g}$ (Nunez, 1978), nombre insuffisant pour jouer un rôle important dans l'ouverture du fromage. L'intérêt technologique des bactéries du genre Leuconostoc dans la fabrication du fromage de Cabrales semble donc négligeable.

Les leuconostocs sont moins sensibles que les streptocoques au chlorure de sodium. Le rapport « temps de génération avec $5 \mathrm{p} .100$ $\mathrm{NaCl} /$ temps de génération avec 0 p. $100 \mathrm{NaCl}$ » était en moyenne de 2,50 pour les souches de l'intérieur et de 3,65 pour celles de l'extérieur. Nous avons constaté pour 11 souches sur 12 une stimulation en présence de 1 p. $100 \mathrm{NaCl}$. L. lactis était la plus halosensible : le rapport mentionné était 4,19 pour une souche de l'intérieur et 5,80 en moyenne pour deux souches de l'extérieur, ce qui peut expliquer la faible proportion des souches isolées.

\section{c) LES LACTOBACiLLES}

Dans le fromage de Cabrales la population de lactobacilles n'arrivait à son maximum, supérieur à $10^{8} / \mathrm{g}$, qu'après $60 \mathrm{j}$ d'affinage (Nunez, 1978) au lieu de $10 \mathrm{j}$ pour le Roquefort (Devoyod, 1970), ce qui s'explique par les différentes dates de salage : $3^{\mathrm{e}}$ à $4^{\mathrm{e}} \mathrm{j}$ pour le Cabrales et $10^{\mathrm{e}}$ à $16^{\mathrm{e}} \mathrm{j}$ pour le Roquefort.

Lactobacillus plantarum était l'espèce la plus courante dans le fromage de Cabrales : elle représentait 82 p. 100 des souches de lactobacilles isolées à la surface et 97 p. 100 de celles isolées au centre du fromage, ce qui contredit les résultats de Burgos et al. (1971). Sharpe et Brindley (1956) ont montré que L. plantarum était également l'espèce dominante à la surface du fromage de Stilton et Devoyod (1970) a signalé cette espèce comme la plus fréquemment isolée dans le fromage de Roquefort.

Les six souches de L. casei subsp. casei étudiées, ainsi qu'une des deux souches de L. casei subsp. rhamnosus, ont été isolées à la surface du fromage après salage.

Le rapport « temps de génération avec 5 p. $100 \mathrm{NaCl} /$ temps de génération avec 0 p. $100 \mathrm{NaCl}$ »pour les lactobacilles était le plus bas des trois genres étudiés. Nous avons observé une stimulation pour 9 souches sur 12 en présence de 1 p. $100 \mathrm{NaCl}$, pour 8 souches sur 12 en présence de 2 p. $100 \mathrm{NaCl}$ et pour 4 souches sur 12 en présence de 3 p. $100 \mathrm{NaCl}$.

La moyenne des temps de génération pour $L$. plantarum en présence de 5 p. $100 \mathrm{NaCl}$ était seulement de $223 \mathrm{mn}$ pour les souches 
de la surface et de $187 \mathrm{mn}$ pour les souches du centre du fromage. A cause de cette halotolérance et de sa capacité à se développer à bas $\mathrm{pH}, L$. plantarum devenait l'espèce bactérienne dominante pendant et après le deuxième mois d'affinage dans le fromage de Cabrales.

En raison de leurs propriétés protéolytiques et lipolytiques, les lactobacilles sont susceptibles de participer à la maturation du fromage de Cabrales : au $60^{\mathrm{e}} \mathrm{j}$, ils représentaient dans l'une des fabrications (A) 78 p. 100 des germes totaux au centre du fromage et 29 p. 100 dans l'autre (B); au $90^{\mathrm{c}} \mathrm{j}$, ces proportions étaient de 85 p. 100 pour la fabrication A et de 48 p. 100 pour la fabrication B.

\section{Rés umé}

Lors d'une étude sur deux fabrications traditionnelles de fromage bleu de Cabrales, 261 souches de bactéries lactiques ont été isolées à partir du lait, du caillé et du fromage pendant la maturation et identifiées comme étant des streptocoques (85), des leuconostocs (59) et des lactobacilles (117).

Parmi les streptocoques, Str. lactis dominait pendant la fabrication et au cours de tout l'affinage ; la plupart des souches étudiées étaient halosensibles et faibles productrices d'acide lactique. La peu nombreuse population d'entérocoques appartenait surtout à l'espèce Str. faecium. L'espèce Str. acidominimus était présente à la surface du fromage affiné.

Leuconostoc dextranicum et L. paramesenteroides représentaient ensemble 76 p. 100 des leuconostocs isolés. Moins sensibles au chlorure de sodium que les streptocoques et ne produisant pas du diacétyle, leur faible nombre leur empêche de jouer un rôle dans l'ouverture de la pâte.

L'espèce dominante de lactobacilles était L. plantarum : 92 p. 100 des souches isolées. A cause de son halotolérance, elle constituait la presque totalité de la flore bactérienne pendant et après le deuxième mois d'affinage pouvant ainsi intervenir dans le mécanisme de la maturation du fromage de Cabrales.

\section{S u m m a ry}

Throughout a work on two batches of traditionally made Cabrales blue cheese, 261 lactic acid bacteria strains were isolated from milk, curd and cheeses at different stages of their ripening period and identified as follows : 85 streptococci, 59 leuconostocs and 117 lactobacilli.

Among streptococci, Str. lactis prevailed during cheese-making and the whole ripening period; most of our strains showed a low 
salt tolerance and were weak lactic acid producers. Enterococci, present at low levels, belonged mainly to the species Str. faecium. The species Str. acidominimus was present on the surface of ripened cheese.

Leuconostoc dextranicum and L. paramesenteroides comprised as a whole 76 p. 100 of the isolated leuconostocs. Less affected by sodium chloride than streptococci and not producing diacetyl, low levels limit their contribution to the opening of the curd.

Accounting for 92 p. 100 of the isolated lactobacilli strains, Lacto. bacillus plantarum was the dominant species. Due to its halotolerance L. plantarum became the majority bacterial flora from the second month of maturation, being probably involved in the process of Cabrales cheese ripening.

Reçu pour publication en juillet 1979.

\section{Bibliographie}

BARRITT (M. M.) (1936), - The intensification of the Voges-Proskauer reaction by the addition of $\alpha$-naphtol. J. Path. Bact., 42, 441.

Buchanan (R.E.) and Gibbons (N. E.) (1974). - Bergey's Manual of Determinative Bacteriology. 8th ed. Waverley Press Inc., Baltimore.

Burgos (J.), Lopez (A.) i Sala-Trepat (F. J.) (1971). - Maduracion del queso "Cabrales". Microflora: I. Lactobacilos. Anales Fac. Vet. Leon, 17, 109.

CavetT (J. J.) and GaRvie (E. I.) (1967), - Biochemical and serological characters of three strains of streptococci previously reported as Streptococcus cremoris isolated from deep-frozen peas after thawing. J. appl. Bact., 30, 377.

De Man (J. C.), Rogosa (M.) and Sharpe (E.) (1960). - A medium for the cultivation of lactobacilli. J. appl. Bact., 23, 130.

Devoyod (J. J.) (1969). - La flore microbienne du fromage de Roquefort. IV. Les entérocoques. Le Lait, 49, 637.

Devoyod (J. J.) (1970). - La flore microbienne du fromage de Roquefort. V. Les lactobacilles. Le Lait, 50, 277.

Devoyod (J. J.) et Desmazeaud (M.) (1970). - Les associations microbiennes dans le fromage de Roquefort. I. Action des entérocoques vis-à-vis des streptocoques lactiques et des leuconostoc. Le Lait, 50, 374.

Devoyod (J. J.) et Muller (M.) (1969). - La flore microbienne du fromage de Roquefort. III. Les streptocoques lactiques et les leuconostoc. Influence de différents micro-organismes de contamination. Le Lait, 49, 369.

Elliker (P. R.), ANDERson (A. W.) and Hannesson (G.) (1956). - An agar culture medium for lactic acid streptococci and lactobacilli. J. Dairy Sci., 39, 1612.

Frazier (W. C.) (1926). - A method for the detection of changes in gelatin due to bacteria. J. infect. Dis., 39, 302.

Garvie (E. I.) (1960), - The genus Leuconostoc and its nomenclature. J. Dairy Res., 27, 283.

Garvie (E. I.) (1967). - Leuconostoc oenos sp. nov. J. gen. Microbiol., 48, 431.

Kenner (B. A.), Clark (H. F.) and Kabler (P. W.) (1961). - Faecal streptococci. I. Cultivation and enumeration of streptococci in surface waters. Appl. Microbiol., 9, 15. 
NAYloR (J.) and Sharpe (M. E.) (1958). - Lactobacilli in Cheddar cheese. I. The use of selective media for isolation and of serological typing for identification. J. Dairy Res., 25, 92.

NunEz (M.) (1976). - Flora microbiana del queso Manchego. V. Leuconostoc. Anales INIA, Ser. General, 4, 67.

NunEz (M.) (1978). - Microflora of Cabrales cheese: changes during maturation. J. Dairy Res., 45, 501.

ORvin-Mundt (J.) (1976). - Streptococci in dried and frozen foods. J. Milk \& Food Tech., 39, 413.

Reddy (M. S.), Vedamuthu (E. R.) and Reinbold (G. W.) (1971). - A differential broth for separating the lactic streptococci. J. Milk \& Food Tech., 34, 43.

SANDine (W. E.), Elliker (P.R.) and Hays (H.) (1962). - Cultural studies on Streptococcus diacetilactis and other members of the lactic streptococcus group. Can. J. Microbiol., 8, 161.

Sharpe (M. E.) and Brindley (M.) (1956). - Lactobacilli isolated from the surface of normal and slipcoat Stilton cheese. J. Dairy Res., 23, 361.

Shatтock (P. M.) and Hirsch (A.) (1947). - Abstracts of Microbiological Methods. J. Path. Bact., 59, 495. Ed. V.B.D. Skerman (1969). Wiley-Interscience, New York. 